\title{
Sorg, Petra (2020). Religionsunterricht im globalisierten Klassenzimmer. Positionierungen von Lernenden im multireligiösen Kontext beruflicher Schulen. Münster: Waxmann. ISBN 978-3-8309-4I33-5. 344 Seiten.
}

\section{Matthias Gronover}

Eberhard Karls Universität Tübingen (matthias.gronover@uni-tuebingen.de)

Wenn es stimmt, dass der Lernort Religionsunterricht an berufsbildenden Schulen als Pionier für die Entwicklung religionspädagogischer Optionen gilt, die der Weiterentwicklung des Religionsunterrichts überhaupt dienen, dann sollte den Entwicklungen dort hohe Aufmerksamkeit zukommen. Die hier zu besprechende Arbeit leistet einen präzisen und methodisch sehr stringenten Beitrag zur Frage der Positionierung von Schülerinnen und Schülern an berufsbildenden Schulen.

Dabei ist die Frage der Bedeutung von Positionalität für religiöse Bildungsprozesse eine zwar in der Religionspädagogik noch diskutierte, für die Verfasserin aber in mehrfacher Hinsicht zentrale Dimension zum Verständnis der Lernprozesse: Zum einen gebe sie Aufschluss über Lernchancen und Lernhindernisse bei Schülerinnen und Schülern, zudem verweisen Positionierungen immer auch auf soziale Hintergründe der Lernenden, und schließlich mündet beides in Kriterien zur Weiterentwicklung religionsdidaktisch Optionen. Insofern ist die Frage nach Positionierungen tatsächlich zentral, wenn man, wie in berufsbildenden Schulen fast durchgängig üblich, religiöse Bildung nicht im Machtgefälle zwischen Lehrenden und Lernenden, sondern auf Augenhöhe mit den meist jungen Erwachsenen versteht und religiöse Bildung mit dem Ziel der religiösen Mündigkeit verfolgt wird.

Wichtige Referenzen für die Studie sind die Theorie der critical incidents nach Joachim Willems und der Ansatz von Mirjam Schambeck zum interreligiösen Lernen, (Nächsten-)Liebe als Medium interreligiöser Lernprozesse. Beide Ansätze werden eng mit dem Vorhaben verwoben und an verschiedenen Stellen diskutiert. Die Entscheidung, die vielfältige theoretische Diskussion auf diese beiden Ansätze zu konzentrieren und mit den eigenen empirischen Ergebnissen ins Gespräch zu bringen, erweist sich als facettenreich und fruchtbar. Warum?

Die Autorin geht in vier Schritten vor. In einem ersten Schritt erhebt sie in einer quantitativen Teilstudie ( $\mathrm{N}=301)$ mittels des Schwarz'schen Wertefeldes, „,welche Werte, welche Einstellungen und Identitätsaktivierungen“ die Lernenden prägen (62). Dieser quantitative Teil orientiert sich jedoch sehr stark am oben genannten Wertefeld und der Frage, inwieweit die Schülerinnen und Schüler ihrem jeweiligen sozialen Milieu verhaftet sind und/oder im Blick auf ihre Identität eher dem Pol Selbststeigerung oder demjenigen der Selbst-Transzendenz zuzuordnen sind. Inhaltliche Fragen zu Zustimmungsgraden zu positionellen Gehalten von beispielsweise Judentum, Islam und Christentum werden in dieser Studie nicht gestellt.

Die qualitative Studie wurde anhand eines Interviewleitfadens durchgeführt, wobei die tatsächliche Form des Interviews „zwischen den Polen narratives und standardisiertes Interview“ (143) zu verorten ist. Ziel war es, das „prozessuale Geschehen im Klassenraum aus Sicht der Lernenden nachzuzeichnen, zu beschreiben und verstehen zu können“ (141). In vorbildlicher Weise wird das Zueinander des quantitativen und qualitativen Teils dieses Forschungsprojekts beschrieben (131-135).

Da die empirische Methodologie der Studie sehr sorgfältig vorgestellt sowie das Ineinander von quantitativer und qualitativer Teilstudie begründet wird („,mixed-methods“) und auch die Analyse der Daten sorgfältig stattfindet, drängen sich umso mehr Fragen der normativen Hintergründe auf. 
Aber zunächst noch zu einigen Erkenntnissen: Zum einen stellt die Verfasserin fest, dass die von den Lernenden präferierten Werte (ihre „Identitätspräferenzen“) das bestimmen, was sie für sich als Lernertrag identifizieren. Dabei ist deutlich zu sehen, dass Lernende, die sehr stark von einer sozialen Gruppe (dem Islam) bestimmt sind, als Lernertrag ,"stärker als alle anderen Gruppen die Bestätigung und Stärkung ihrer bisherigen Sicht" (107) betonen. Die innere Logik der Forschung mit dem oben genannten Wertefeld bringt es mit sich, dass analog zum Ergebnis mit Blick auf die Verbundenheit zur eigenen, sozialen Gruppe, diejenigen Lernende, die „eher der personalen Identität zusprechen, Methoden bevorzugen, die eher auf individuelles Erkennen und Verstehen und Entdecken, kurz auf Neues fokussieren“ (115).

Im qualitativen Teil zeigt die Autorin, was die soziale Raumtheorie für die Analyse und Interpretation ihrer Interviews bieten kann. Dabei kontrastiert sie Erfahrungen der Interviewten außerhalb des Klassenraums mit Erfahrungen im Klassenraum und kann so die Positionierungsprozesse dieser Individuen nachzeichnen. Durch die Kontrastierung wird bezogen auf die Interviewten deutlich, wie bestimmte Einstellungen und Haltungen aus deren Sicht begründet werden und die Vielfalt der Positionierungen wird nachvollziehbar. So zeigt das Interview mit Aleida (223-231), wie der frühere Schulraum sich analytisch mit dem präsentischen Klassenraum in Beziehung setzen lässt und wie Positionierung innerhalb des Kontrasts, der sich dadurch abzeichnet, geschieht: Im früheren Klassenraum war ihre Religion für Aleida für ihr Selbstverständnis nicht zentral. In der neuen Klasse der Fachoberschule allerdings, mit muslimischen Mitschülern, positioniert sie sich als Christin und grenzt sich von muslimischen Jungen ab (231). In der religiösen Heterogenität des neuen Lernumfeldes wird Aleida sich ihres christlichen Hintergrunds und - vor allem - dessen Bedeutung für ihr Selbstverständnis bewusst.

Insgesamt und über beide Teilstudien hinweg zeigt die Autorin, „, dass das Produktivmachen des Fremden nur Lernenden mit bevorzugter personaler Identität zugänglich war, während es für die anderen kaum Erkenntnisgewinn bringen konnte“ (241). Außerdem zeigt sie, „warum im Klassenraum als sozialem Gefüge es den jungen Männern offenbar weniger gelingt, Neues zu integrieren - im Gegensatz zu den jungen Frauen“ (236). Das Verhaftetsein in der eigenen sozialen Gruppe und das Bedürfnis, von ihr auch Bestätigung zu bekommen, stehe einer erkenntnisförderlichen Offenheit gegenüber Neuem im Weg.

Im letzten Teil der Studie formuliert die Autorin didaktische Konsequenzen, die sich stark auf den Aspekt der Individualität der Lernenden konzentrieren. Hier kommen die Ansätze von Mirjam Schambeck (Liebe als Medium in interreligiösen Lernprozessen) und Joachim Willems (dessen Weiterarbeit an der Theorie der critical incidents) ins Spiel. Mit Blick auf Schambeck konstatiert die Autorin, dass bei ihr der positionelle Gehalt einer Religion mit der Positionalität der Schülerinnen und Schüler zusammenfalle. Dagegen stellt die Autorin fest, dass sich das Eigene der Religion der Schülerinnen und Schüler ja gerade im Freiraum zeige, "der sich in der Wahrnehmung von Differenzen in der Narration des Eigenen ergibt" (288). Eng damit zusammen hängt die Kritik an Willems, die sich aus vier Gründen speist: Erstens seien critical incidents vor allem für Lernende gut, deren personale Identität aktiviert werde; zweitens bringe die Offenheit der Problemstellung von critical incidents immer auch ein gewisses Rätselraten mit sich; drittens spielten critical incidents immer mit stereotypen Zuschreibungen, beispielsweise wenn es um „, die“ Muslime gehe; und viertens bestehe die Gefahr, dass sich die sozialen Identitäten der Lernenden gegeneinander positionierten (290).

So stringent die Studie durchgeführt ist, so grundsätzlich kann man fragen, ob die Zuspitzung sowohl des empirischen Teils als auch der Formulierung der didaktischen Konsequenzen notwendig die Individualität der Lernenden ins Zentrum rücken muss. Auch in Hessen, wo die Studie durchgeführt wurde, findet religiöse Bildung in berufsbildenden Schulen in einem konfessionellen Religionsunterricht statt. Aber wie verhält sich dieser konfessionelle Rahmen zur religiösen Heterogenität in der Klassenzusam- 
mensetzung? Welche Orientierungen müssen in den Blick genommen werden, wenn man beispielsweise die Ergebnisse der Studie zur personalen und sozialen Identität der Lernenden ernst nimmt? Die Studie erweckt den Eindruck, als erschöpfe sich Religion aus den individuellen Vorstellungen der Adressaten religiöser Bildung.

Der Begriff der religiösen Heterogenität, durch den die Diskussion um Positionalität erst an Fahrt gewinnt, bleibt in der Studie deutlich unterbestimmt und hinter der religionspädagogischen Diskussion zurück. Zwar wird deutlich, wie die Autorin Positionierungen versteht und ihre Ausführungen liest man mit großem Erkenntnisgewinn. Gleichzeitig wäre die Diskussion um positionelle und kontextuelle sowie stilistische und biografische Differenzen anhand des Datenmaterials der Studie wichtig, um das Verständnis von Heterogenität zu vertiefen und in den sich darin zeigenden Brüchen eine Vorstellung vom "globalisierten Klassenzimmer" sehen zu können. Eine Einordnung des Begriffs der Positionalität in diese Diskussion wäre hilfreich, zumal auch der von der Autorin vorgetragene offene Begriff der Positionalität ein bestimmtes Verständnis von individualisierter Religion mit sich bringt.

Außerdem ist zu fragen, ob die Erkenntnisse der berufsorientierten Religionspädagogik zu religionsdidaktischen Fragestellungen, die Konsequenzen der Autorin im Blick auf den Religionsunterricht als safe space, das Begegnungslernen, die Dekonstruktion von Zuschreibungen und vor allem der Perspektivenwechsel noch weiter hätten konkretisiert werden können.

Insgesamt handelt es sich um eine Studie, der eine breite Lesegemeinschaft zu wünschen ist. Für die berufsorientierte Religionspädagogik ist sie eine große Bereicherung. 